\title{
A Narrative Review of Clinical Decision Support for Inpatient Clinical Pharmacists
}

\author{
Liang Yan ${ }^{1}$ Thomas Reese ${ }^{2}$ Scott D. Nelson ${ }^{3}$ \\ 1 University of Utah College of Pharmacy, University of Utah Health, \\ Salt Lake City, Utah, United States \\ ${ }^{2}$ Department of Biomedical Informatics, University of Utah School of \\ Medicine, Salt Lake City, Utah, United States \\ ${ }^{3}$ Department of Biomedical Informatics, Vanderbilt University \\ Medical Center, Nashville, Tennessee, United States
}

\author{
Address for correspondence Liang Yan, PharmD, University of Utah \\ College of Pharmacy, University of Utah Health, 30 South 2000 E, \\ Salt Lake City, UT 84112, United States \\ (e-mail: Liang.Yan@pharm.utah.edu).
}

\section{Abstract}

Keywords

- clinical decision support

- pharmacy information systems

- inpatient care

- pharmacy

- clinical informatics

- pharmacist
Objective Increasingly, pharmacists provide team-based care that impacts patient care; however, the extent of recent clinical decision support (CDS), targeted to support the evolving roles of pharmacists, is unknown. Our objective was to evaluate the literature to understand the impact of clinical pharmacists using CDS.

Methods We searched MEDLINE, EMBASE, and Cochrane Central for randomized controlled trials, nonrandomized trials, and quasi-experimental studies which evaluated CDS tools that were developed for inpatient pharmacists as a target user. The primary outcome of our analysis was the impact of CDS on patient safety, quality use of medication, and quality of care. Outcomes were scored as positive, negative, or neutral. The secondary outcome was the proportion of CDS developed for tasks other than medication order verification. Study quality was assessed using the Newcastle-Ottawa Scale.

Results Of 4,365 potentially relevant articles, 15 were included. Five studies were randomized controlled trials. All included studies were rated as good quality. Of the studies evaluating inpatient pharmacists using a CDS tool, four showed significantly improved quality use of medications, four showed significantly improved patient safety, and three showed significantly improved quality of care. Six studies (40\%) supported expanded roles of clinical pharmacists.

Conclusion These results suggest that CDS can support clinical inpatient pharmacists in preventing medication errors and optimizing pharmacotherapy. Moreover, an increasing number of CDS tools have been developed for pharmacists' roles outside of order verification, whereby further supporting and establishing pharmacists as leaders in safe and effective pharmacotherapy.

\section{Background and Significance}

Pharmacists' roles have expanded from traditional medication verification and dispensing to team-based patient care. ${ }^{1,2}$ Since the early 1970s, pharmacists have been tangentially involved with inpatient care through pharmacokinetic consultations. More recently, pharmacists are assuming providers' roles with collaborative practice agreements, which in some cases allow them to manage chronic health care conditions, such as diabetes and cardiovascular conditions. ${ }^{1}$ Clinical pharmacists' roles are becoming more specialized through pharmacy residency training programs and specialty certification, and they are recognized as crucial members of the multidisciplinary health received

August 25, 2020

accepted after revision

December 14, 2020 (c) 2021. Thieme. All rights reserved. Georg Thieme Verlag KG,

Rüdigerstraße 14,

70469 Stuttgart, Germany
DOI https://doi.org/

10.1055/s-0041-1722916. ISSN 1869-0327. 
care team. ${ }^{3}$ As with providers, clinical pharmacists can be supported by health information technology, which has been considered a major factor in preventing medication errors and patient harm. ${ }^{2}$

Electronic health record (EHR) adoption and clinical decision support (CDS) implementation have also expanded in recent years. ${ }^{4}$ As of 2017, $94 \%$ of U.S. hospitals used an EHR system. ${ }^{4} \mathrm{CDS}$ has been characterized as a health information technology that provides patient-specific information that is intelligently filtered and presented at appropriate times for decision making. ${ }^{5-7} \mathrm{CDS}$ can be developed and refined through clinician observations, suggestions, and preferences. ${ }^{8-10}$ Some examples of CDS include passively providing clinicians with helpful information without interrupting their workflow or process, such as order facilitators (e.g., order sets and default settings), relevant information displays (e.g., info-buttons and links to additional resources), and even in-line displays of information (e.g., allergy or dose alerts). ${ }^{11}$ CDS also includes active or interruptive alerting, such as hard-stop alerts and reminders. Unfortunately, even with more EHRs and CDS, deaths from medication errors still occur at an alarming rate. ${ }^{12}$

Pharmacists play a crucial role in identifying and preventing medication errors and CDS tools can help improve pharmacists' proficiency. A study found that $64 \%$ of prescribing errors could be prevented with pharmacists and CDS. The authors concluded that pharmacists' involvement is crucial for achieving maximum medication safety. ${ }^{1}$ Moreover, systematic reviews have examined the impact of CDS on a broad range of pharmacy services, demonstrating the benefit of combining pharmacists and CDS. ${ }^{13,14}$ Pharmacists not only use the EHR and CDS, but they also contribute to their design and implementation. ${ }^{15}$ By including pharmacists in CDS design and implementation, the performance and reliability can be increased. ${ }^{16}$
With pharmacists assuming more clinical roles and more CDS tools being developed to support those roles, there is a need to examine the impact of CDS on clinical pharmacy practice. In this study we present a review of the literature regarding the impact of CDS and how it has evolved with clinical pharmacists. We anticipated that inpatient pharmacists using CDS will improve outcomes and that recent CDS supports pharmacists' roles beyond medication verification and dispensing (-Fig. 1).

\section{Methods}

The study protocol was registered in PROSPERO (CRD420150 16952). ${ }^{17}$ We conducted a review of published studies to evaluate the impact of the CDS tools targeted at pharmacists in the inpatient setting. We aligned this review with the PRISMA guidelines and methods from the Cochrane Handbook. $^{18}$

\section{Search Strategy and Study Eligibility}

Working with a medical librarian, we identified cohort studies, observational studies, randomized control trials, and quasiexperimental studies, by searching MEDLINE, EMBASE, and Cochrane Central between January 2009 and October 2020. The search strategy was developed for MEDLINE using PubMed and iteratively refined to capture preidentified studies ( - Table 1 ). We combined keywords, headings, and other search strategies to develop search terms. Once the search strategy was refined with PubMed, we adapted to the other databases. Study inclusion criteria are provided in - Table 2 . Studies were excluded if there was no computerized system involved; the computerized system was not designed with pharmacists as the intended recipients; or the study was not in English. We used Covidence (Melbourne, Australia) to screen studies. ${ }^{19}$ Two reviewers

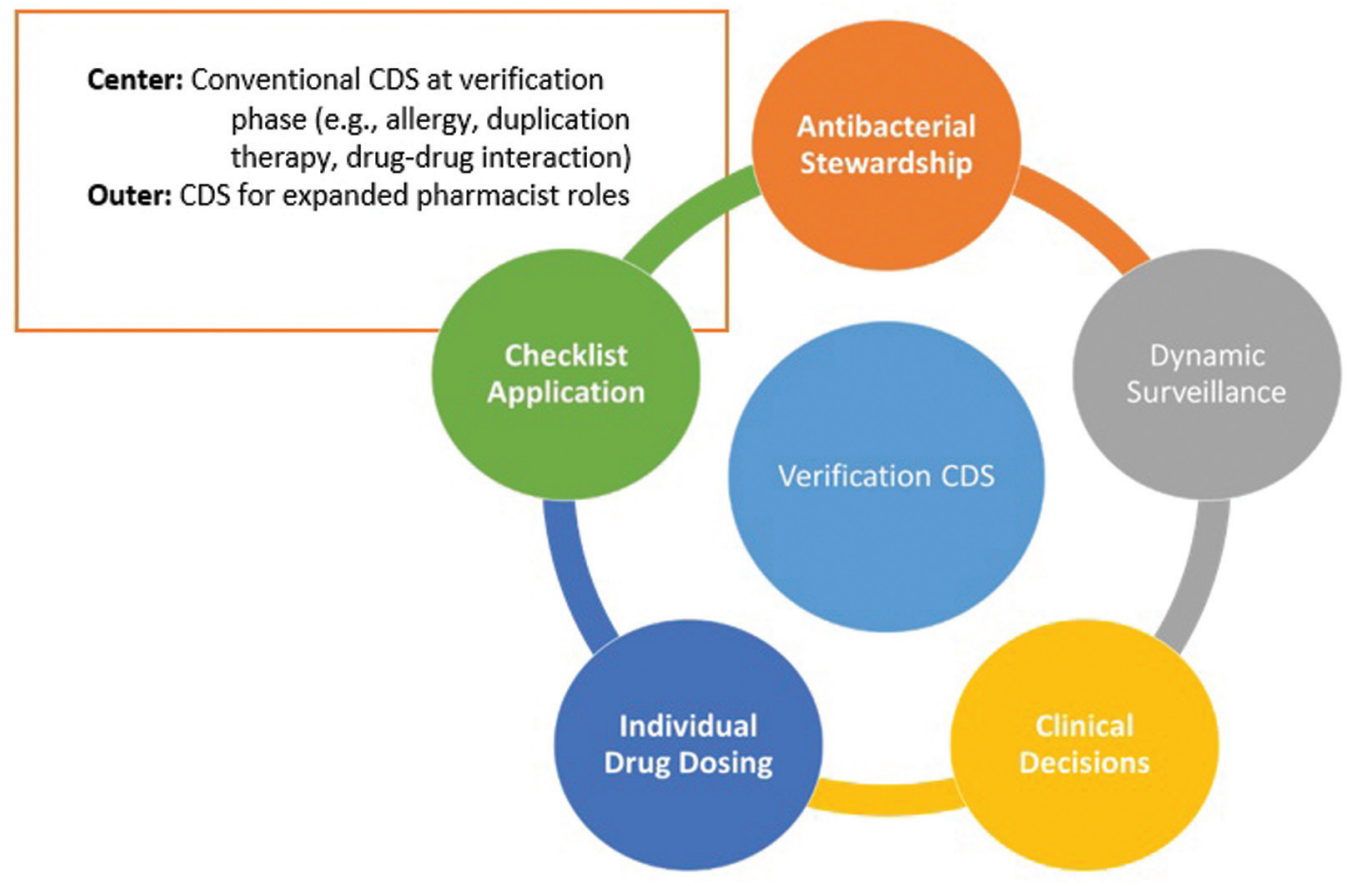

Fig. 1 Expanding roles of pharmacists. 
Table 1 Pharmacy CDS review: PubMed search strategy

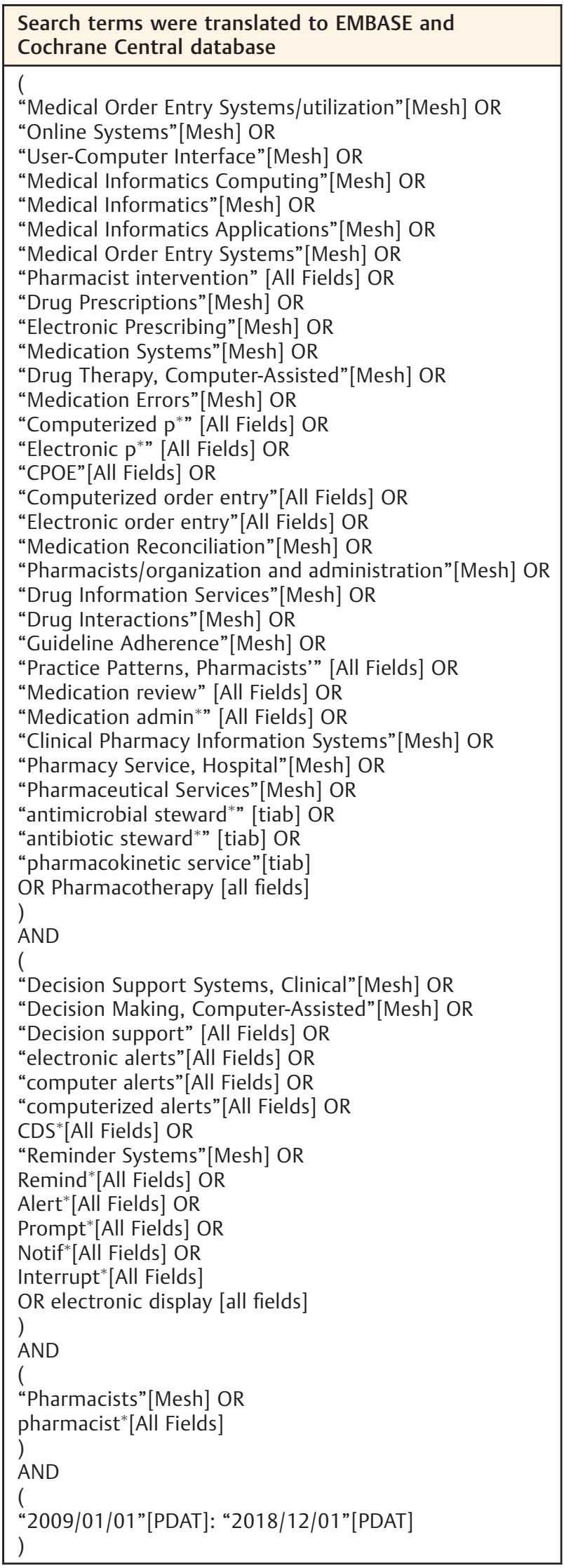

Abbreviation: CDS, clinical decision support. independently screened title/abstracts and full texts and a third reviewer resolved any disputes.

\section{Quality Assessment}

Since the included studies had different designs and outcomes, we used the Newcastle-Ottawa Scale to assess study quality. ${ }^{20}$ Two reviewers independently assessed each included study. Additionally, it was noted whether the study conducted proper statistical analyses and whether the authors had mentioned possible contamination of the study groups.

\section{Reporting and Analysis}

Outcomes were grouped into three main categories: quality use of medication, drug and pharmacotherapy safety, and quality of care. Quality use of medication was defined as the accuracy of medication use and proper management of disease, such as appropriate dosing of medications. Drug and pharmacotherapy safety was defined as the safety of medication use, which was demonstrated by avoiding adverse events or death. Quality of care was defined as patient outcomes, which included parameters such as patient satisfaction and hospital length of stay.

The impact of the CDS intervention was characterized by assigning labels for each study result: " + " study favored the CDS intervention; “- " study favored the control group; "0" was no difference between the intervention and control groups; and "NS" the study did not conduct any statistical analysis or that there was no statistically significant data reported for the specified outcome.

We reported outcome data on whether studies demonstrated at least one positive outcome (i.e., general trend in favor of CDS for a prescribing, clinical, or patient outcome) and statistically significant improvements in favor of CDS on greater than or equal to $50 \%$ of outcomes. We chose to report trends as well as significant results given the likelihood that some studies were underpowered to detect statistically significant differences in outcomes. The outcome data are summarized in a narrative manner instead of a meta-analysis due to the heterogeneity of the study methodologies, comparison groups, intervention targets, and results.

\section{Results}

The search resulted in 4,365 articles. Based on title/abstract screening, 90 articles were selected for full-text review. Fifteen studies met the inclusion criteria (-Fig. 2). Most excluded studies were primarily focused on CDS used by providers and implementations outside the inpatient settings. Key features of the included studies are shown in - Table $3 .^{20-34}$

\section{Study Quality}

Of the 15 included studies, 5 were randomized controlled trials, ${ }^{27,30,31,33,34} 6$ were quasi-experimental, ${ }^{22,25,28,29,32,35}$ 2 were observational, ${ }^{23,26}$ and 2 were cohort studies. ${ }^{21,24}$ All of the studies had a control group and all 15 studies were deemed as good quality using the Newcastle-Ottawa Scale. 
Table 2 Study inclusion criteria

\begin{tabular}{|l|l|}
\hline P: participant/population & Studies listing pharmacists among recipients of the CDS system in the inpatient setting. \\
\hline I: intervention & $\begin{array}{l}\text { The described CDS system had to provide patient-specific information and/or generate } \\
\text { information in an electronic format. }\end{array}$ \\
\hline C: comparator/control & $\begin{array}{l}\text { A comparison of performance of the CDS to routine care or other CDS or computerized } \\
\text { prescriber order entry (CPOE) systems. }\end{array}$ \\
\hline O: outcome & $\begin{array}{l}\text { The primary outcome of the study had to include patient care, clinician effect, } \\
\text { or processes of care. The studies also had to report measured or quantifiable outcomes, } \\
\text { such as cost, patient outcomes, adverse drug events, clinical interventions, etc. }\end{array}$ \\
\hline
\end{tabular}

Abbreviation: CDS, clinical decision support.
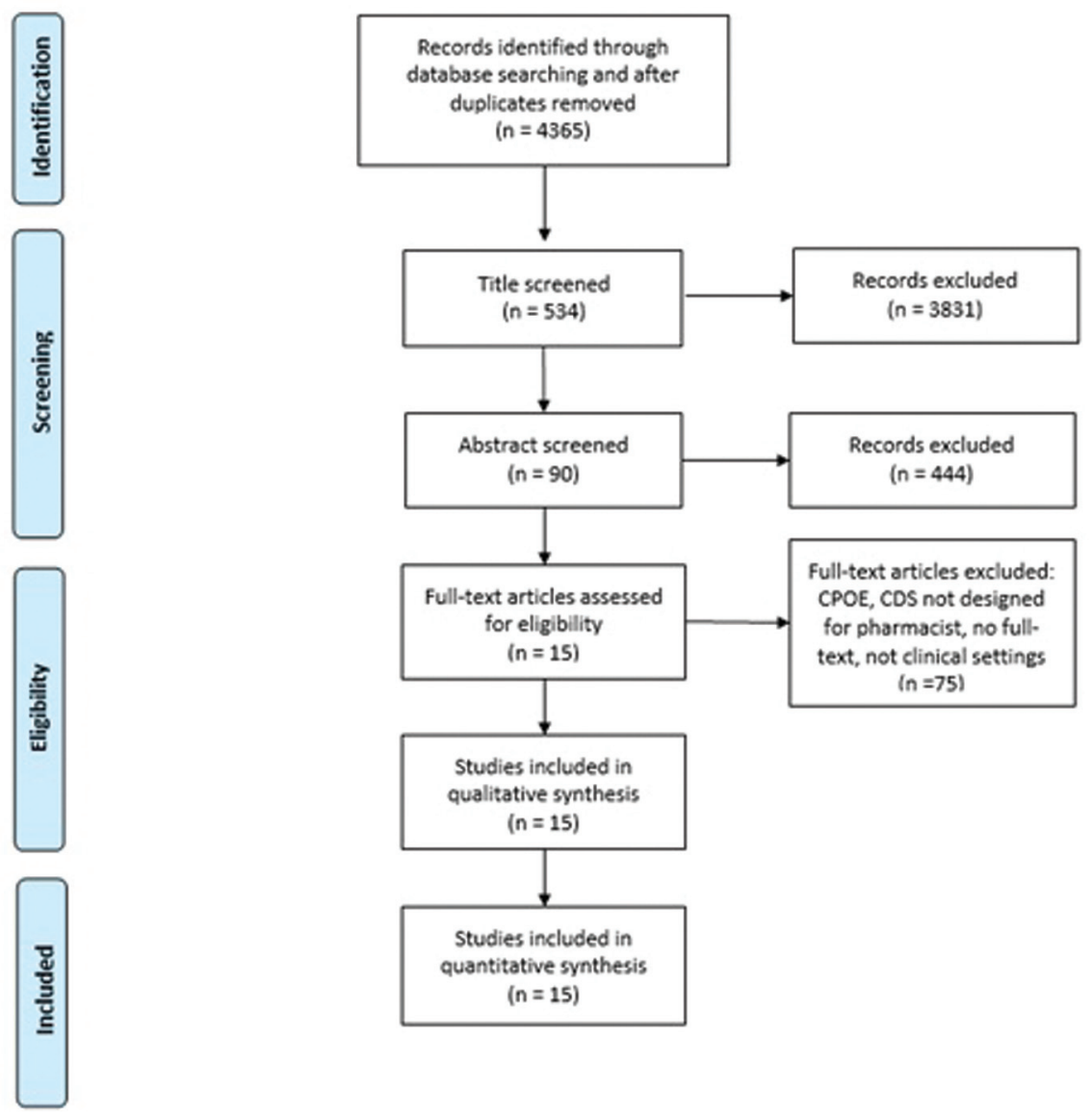

Fig. 2 PRISMA diagram.

\section{Study Characteristics}

Study outcomes were analyzed in three main groups, including quality use of medication, drug and pharmacotherapy safety, and quality of care ( - Table 3 ). Four studies examined the quality use of medications, ${ }^{23,27,31,35}$ seven studies looked at drug and pharmacotherapy safety, 22,24,25,29,30,33,34 and five studies evaluated the quality of care. ${ }^{21,25,26,28,32}$ One study qualified as both drug and pharmacotherapy safety and quality of care. Pharmacists interacted directly with patients in one study through medication reconciliation ${ }^{32}$ and all other CDS systems did not have pharmacist-patient interactions: most of the data were based on measured laboratory results and recorded patient information by nurses and laboratory technicians.
Pharmacists were warned of potential errors through CDS alerting in 11 of the studies. For example, two studies had alerts that interrupted a pharmacist's workflow when a patient had an abnormal serum creatinine. ${ }^{30,34}$ These alerts were designed to prevent medication-caused acute kidney injury and therefore required the pharmacist's immediate attention. None of the included studies discussed alert fatigue or workflow disruption.

Other CDS interventions provided support without interrupting pharmacists' workflow. For example, two studies examined a CDS system that calculated the dose of antibiotics for the pharmacist. ${ }^{23,35}$ Another study evaluated a CDS system that helped identify medication reconciliation errors. ${ }^{32}$ Finally, one study used CDS to examine cost-benefit analysis of medication use. $^{27}$ 
Review of Clinical Decision Support Yan et al. 203

\begin{tabular}{|c|c|c|c|c|c|c|c|c|c|c|}
\hline 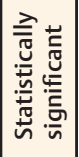 & $\tilde{z}$ & $\tilde{z}$ & $\Sigma$ & 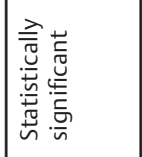 & 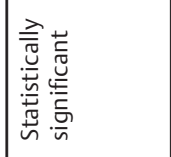 & 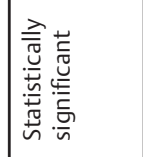 & 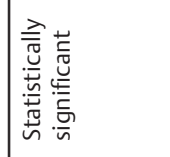 & 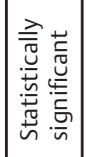 & $\tilde{z}$ & 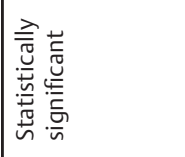 \\
\hline 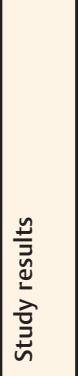 & 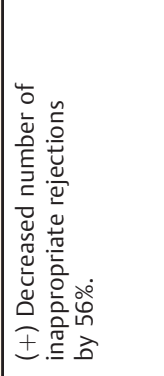 & 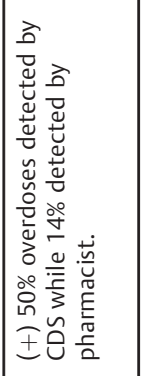 & 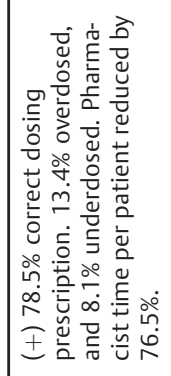 & 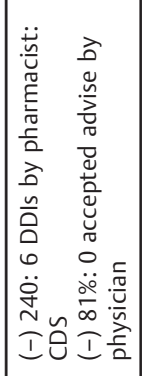 & 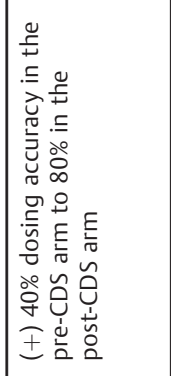 & 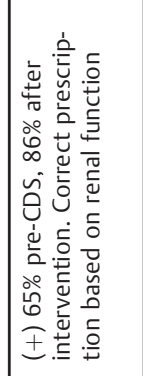 & 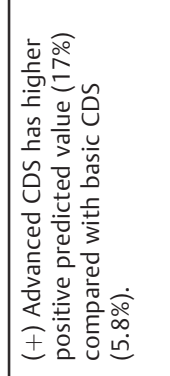 & 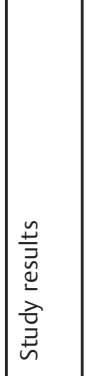 & 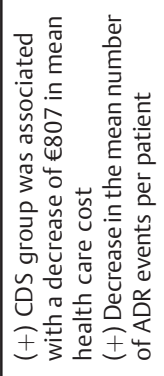 & 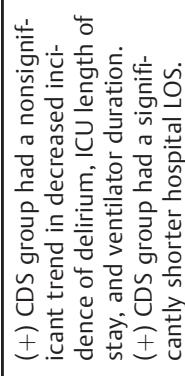 \\
\hline 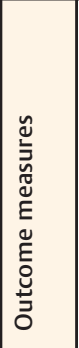 & 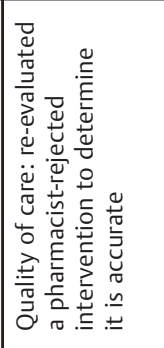 & 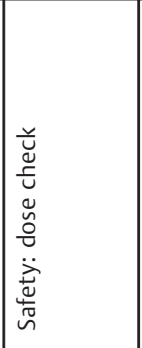 & 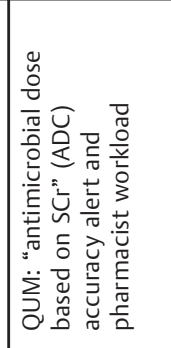 & 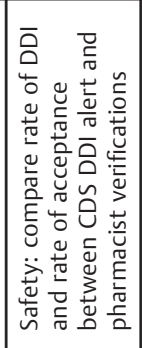 & 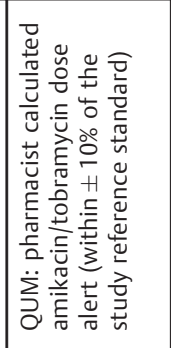 & 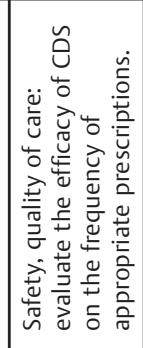 & 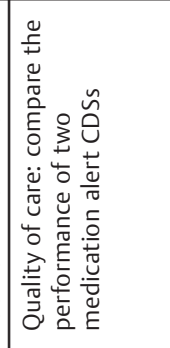 & 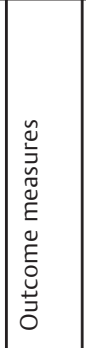 & 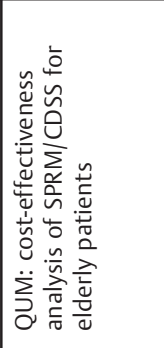 & 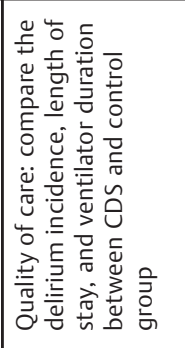 \\
\hline 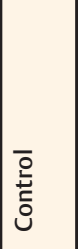 & 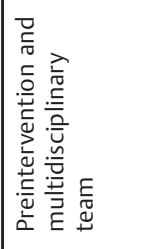 & 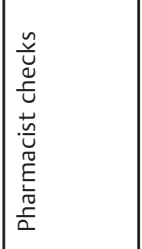 & 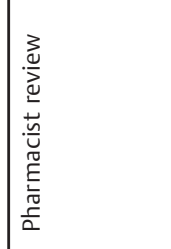 & 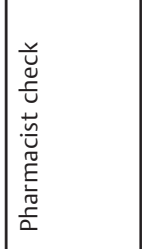 & 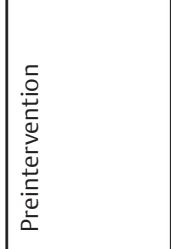 & 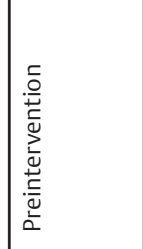 & 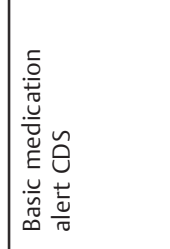 & 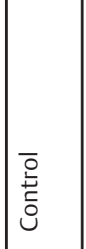 & 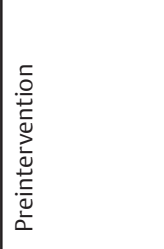 & 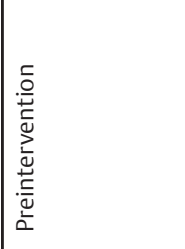 \\
\hline & 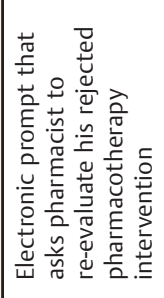 & 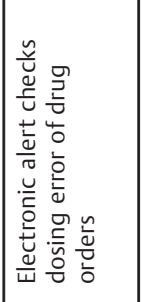 & 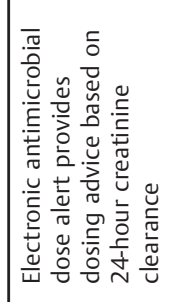 & 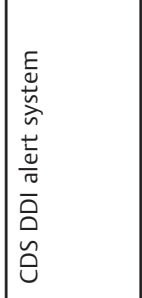 & 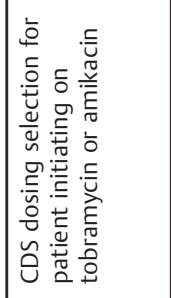 & 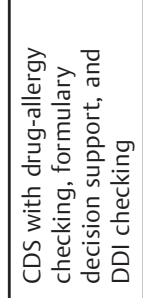 & 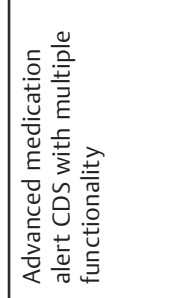 & 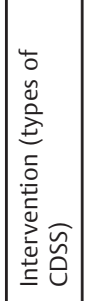 & 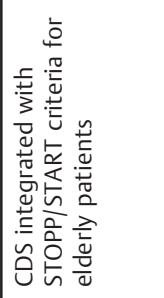 & 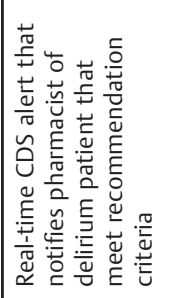 \\
\hline & 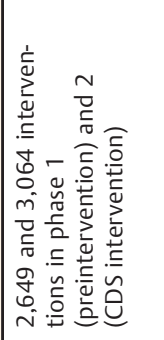 & 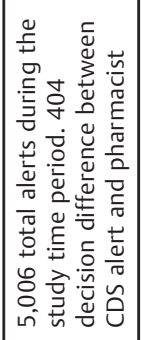 & 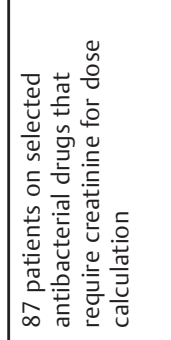 & 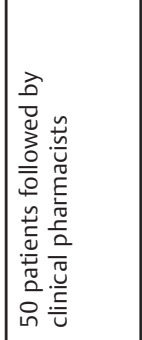 & 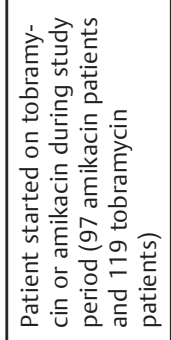 & 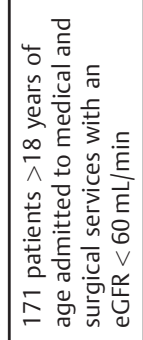 & 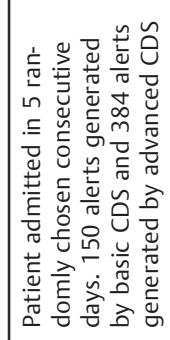 & 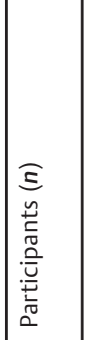 & 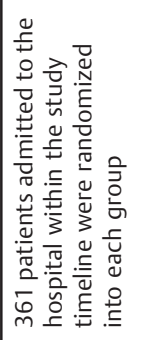 & 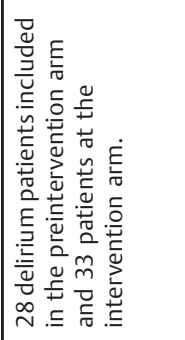 \\
\hline 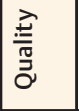 & రั & $\begin{array}{l} \\
\\
o\end{array}$ & 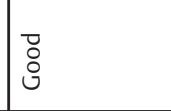 & 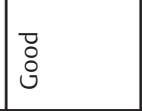 & 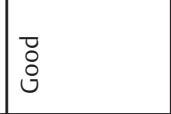 & $\begin{array}{l}\overrightarrow{0} \\
\stackrel{\circ}{\circ} \\
\end{array}$ & \begin{tabular}{|l} 
\\
\\
\\
\end{tabular} & 语 $\frac{\vec{z}}{\vec{v}}$ & 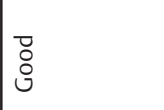 & 离 \\
\hline 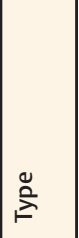 & 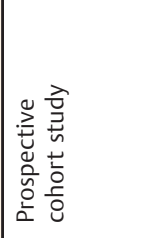 & 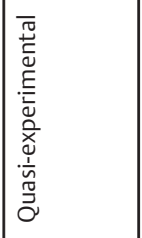 & 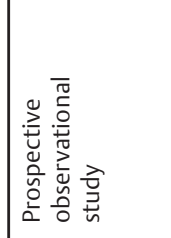 & 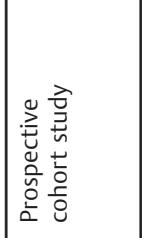 & $\underset{\approx}{\check{z}}$ & 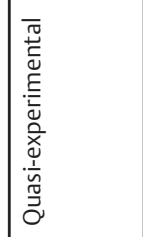 & 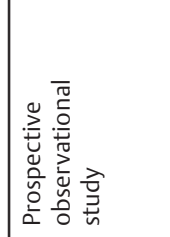 & $\bigwedge_{\gtrless}^{\stackrel{2}{\gtrless}}$ & 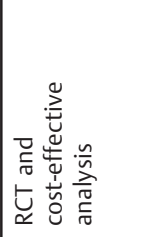 & 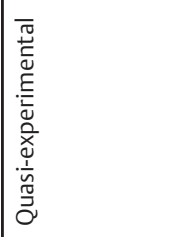 \\
\hline 总 & \begin{tabular}{|l}
$\bar{T}$ \\
$\bar{U}$ \\
$\bar{N}$ \\
$\bar{N}$
\end{tabular} & 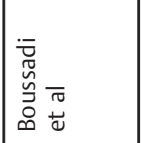 & $\begin{array}{l}\bar{\pi} \\
\tilde{0} \\
\tilde{0} \\
\tilde{U} \\
\tilde{U}\end{array}$ & 离 $\bar{\circlearrowleft}$ & \begin{tabular}{|l}
$\bar{r}$ \\
$\tilde{u}$ \\
$\widetilde{0}$ \\
0
\end{tabular} & $\begin{array}{l}\bar{T} \\
\tilde{D} \\
\tilde{N} \\
\tilde{\sigma}\end{array}$ & 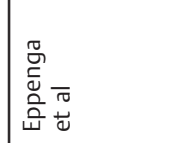 & 效 & 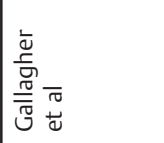 & 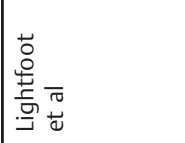 \\
\hline
\end{tabular}




\begin{tabular}{|c|c|c|c|c|c|c|}
\hline 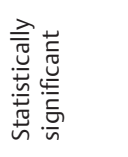 & $\tilde{z}$ & 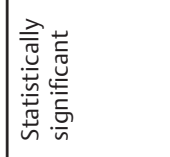 & 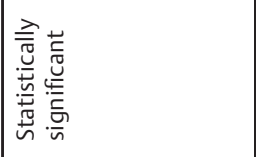 & 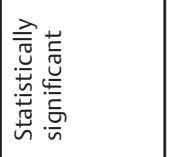 & 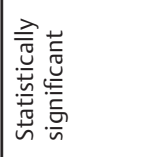 & 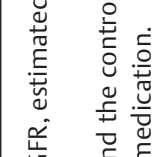 \\
\hline 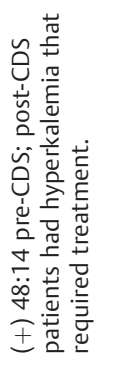 & 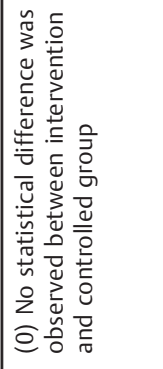 & 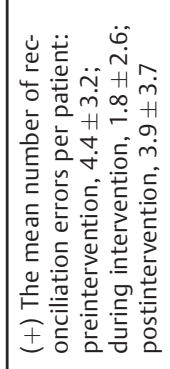 & 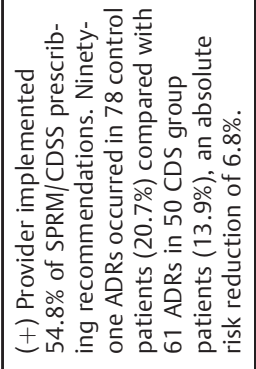 & 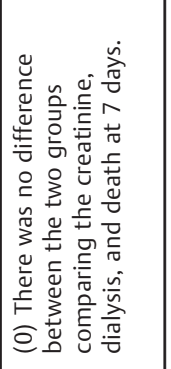 & 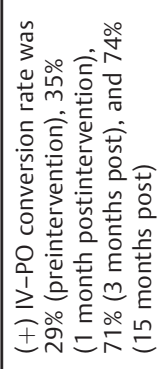 & 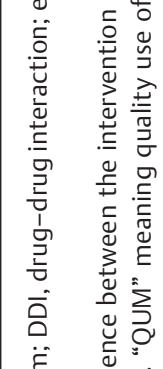 \\
\hline 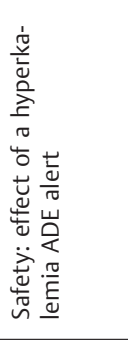 & 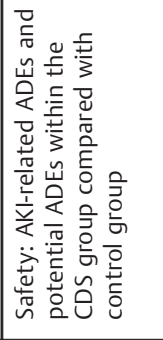 & 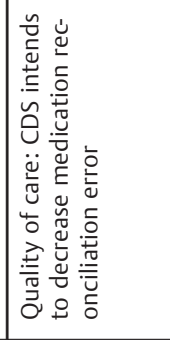 & 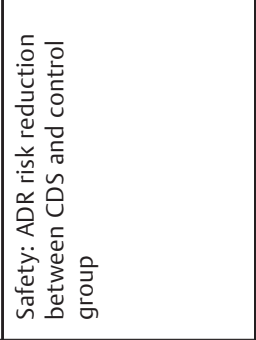 & 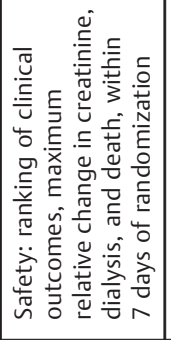 & 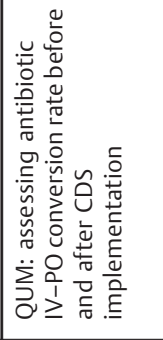 & 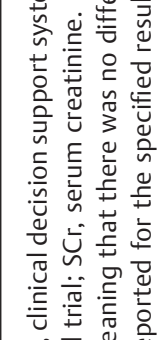 \\
\hline & 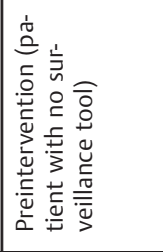 & 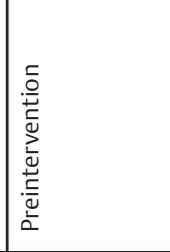 & 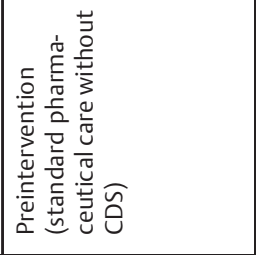 & 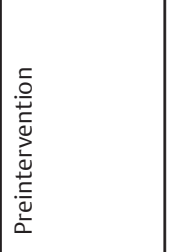 & 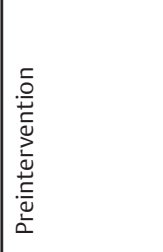 & 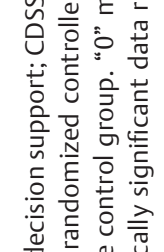 \\
\hline 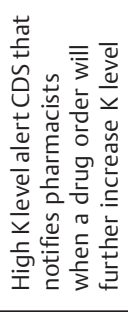 & 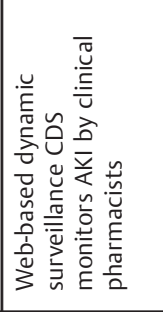 & 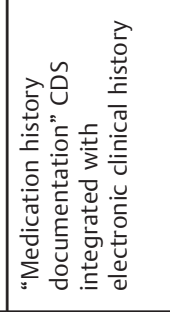 & 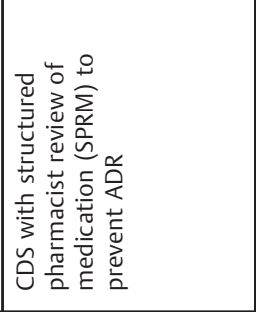 & 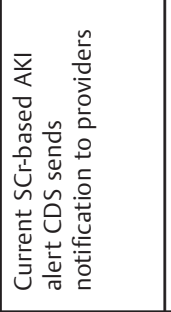 & 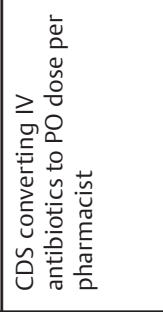 & 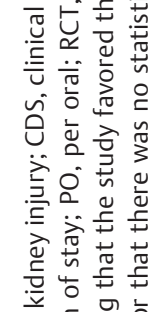 \\
\hline 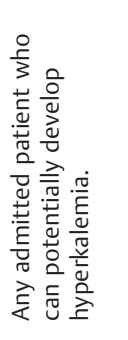 & 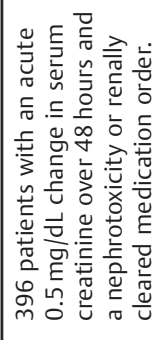 & 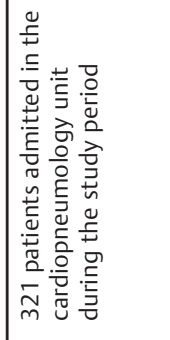 & 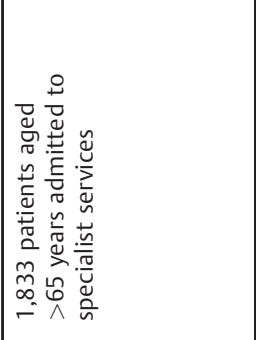 & 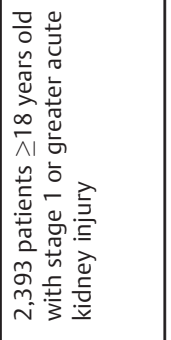 & 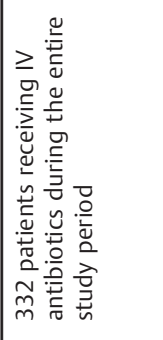 & 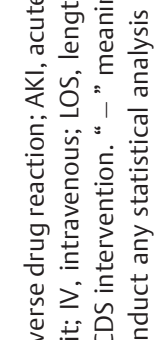 \\
\hline $\begin{array}{l}\text { ¿্口 } \\
\text { ô }\end{array}$ & 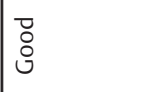 & $\begin{array}{l}\text { ㅁ } \\
\text { o }\end{array}$ & $\begin{array}{l}: \\
\circ \\
o\end{array}$ & 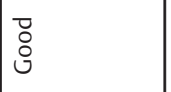 & $\begin{array}{l}\overline{0} \\
\dot{0} \\
\end{array}$ & 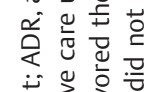 \\
\hline 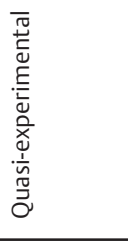 & $\underset{\longleftarrow}{\breve{~}}$ & 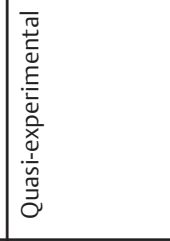 & 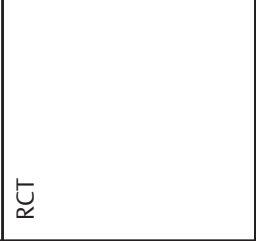 & & 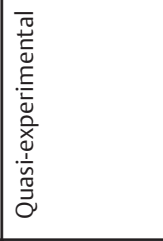 & 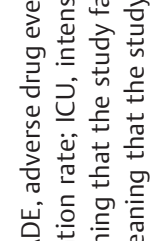 \\
\hline 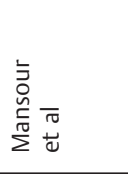 & 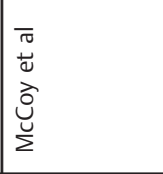 & 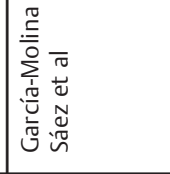 & 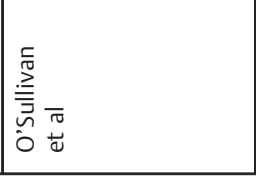 & 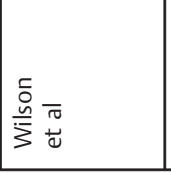 & 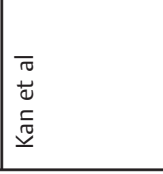 & 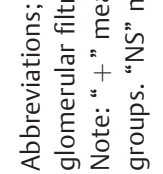 \\
\hline
\end{tabular}




\section{CDS Intervention Analysis}

Eight studies significantly favored the CDS interven$\operatorname{tion}^{25,26,28,29,31-33,35} ; 20 \%$ of the studies presented results that favored the CDS intervention without statistical significance $^{21,23,27} ; 13 \%$ of the studies favored the control groups $^{22,24}$; and $13 \%$ of the studies showed that the CDS interventions had no difference compared with the control group. ${ }^{30,34}$ One study ${ }^{24}$ favors pharmacists' check over CDS; however, this was explained by the authors that the CDS was self-developed and lacks a high level of specificity for drug-drug interactions (DDIs).

CDS interventions covered a wide range pharmacists' practice, including dynamic monitoring, drug safety in low kidney function patients, medication safety in patients with QT prolongation, dosing calculation, medication reconciliation, and general medication use and safety. Eleven of the 14 studies examined CDS application outside medication verification. ${ }^{21,23,27-35}$ Among the 11 studies, $82 \%$ presented results that favored the CDS intervention with statistical significance, ${ }^{21,23,27-29,31-33,35} 18 \%$ of the studies showed that the CDS interventions had no difference compared with the control group, ${ }^{30,34}$ and no study favored the control group.

\section{Discussion}

This study demonstrated that CDS interventions developed for clinical pharmacists can improve health care outcomes. Notably, all studies that evaluated quality use of medication and quality of care showed improved outcomes. Moreover, CDS seems to be evolving with clinical pharmacists' roles, since the majority of studies evaluated an intervention outside order verification tasks.

When compared with CDSs in other settings, studies conducted in community pharmacy practice examined workflow consequences, interactions with patients more frequently, and relied less on EHR information, such as laboratory results. ${ }^{15,36}$ Previous studies have examined CDS that supports prescribing in community and hospital pharmacy settings. ${ }^{13,36}$ These studies concluded that workflow disruption and alert fatigue were major issues causing pharmacists to ignore CDS alerts and prompts. In the present review, we did not identify or conclude that workflow or alert fatigue was a problem. The lack of alert fatigue discussion in the included studies may be the result of the inpatient setting where patient data can be used to refine alert triggers, making alerts more specific. However, we do believe that alert fatigue is still an issue for pharmacists in the inpatient setting given the amount of CDS. ${ }^{37}$

One of the aims of this review was to examine how CDS is meeting the need of clinical pharmacists providing patient care. We found the majority of CDS interventions still focus on drug dosing, DDIs, and identifying or preventing adverse effects; however, we did find CDS interventions that have expanded to other clinical areas, such as managing acute kidney injury, and managing cardiovascular diseases. Also, CDS interventions supported specialized services in the emergency department, geriatric wards, trauma units, and other units. ${ }^{9-11,15-19,21,22}$ These findings suggest that CDS is supporting specialized and decentralized pharmacists' roles in a variety of clinical domains in hospital departments.

Concerns remain on whether advanced CDS systems will replace pharmacists and reduce the number of pharmacy jobs. Based on the included studies, this does not seem to be the case as the majority of CDS interventions were basic alerts and prompts. Two studies used CDS to calculate antimicrobial dosing, but the ultimate decision was still based on pharmacists' discretion. There were no signs that implementation of CDS could replace clinical pharmacists, rather it is more likely that CDS is helping pharmacists increase their efficiency and patient safety. ${ }^{38}$

Limitations of this study should be noted. First, we were unable to conduct meta-analysis due to the heterogeneity of included studies. Second, we only queried studies published in three databases, which means there could be other published (or unpublished) studies that were not included. Finally, the majority of the studies showed a benefit from using CDS, which suggests publication bias may be present.

\section{Conclusion}

With more than $73 \%$ of studies examining CDS outside medication verifications and $82 \%$ of studies favoring the intervention, these findings suggest CDS implementations targeted toward clinical pharmacists have been increasing and improving as pharmacists' roles are becoming more clinical and patient-orientated. We anticipate that as the clinical pharmacy's role expands into more specialized health care fields, CDS interventions will also expand to support pharmacists and improve patient care.

\section{Clinical Relevance Statement}

This review showed that between 2009 and 2020, multiple studies examined clinical pharmacist related nonverification CDS and showed positive results that the CDS facilitates pharmacist's workflow in clinical settings. These CDSs were developed to accommodate the expanding roles of pharmacists. This review has indicated continuous progress for pharmacists to contribute in clinical patient care and makes pharmacist an important member in medical interdisciplinary team.

\section{Multiple Choice Questions}

1. Which of the following pharmacists' roles can be best facilitated by inpatient CDS systems (up to 2)?

a. Pharmacists supervise the medicine supply chain.

b. Pharmacists counsel patients on safe and effective medicine use.

c. Pharmacists advise other health care professionals about safe and effective medicine use.

d. Pharmacists monitor patient's health conditions and determine safety and efficacy of their current medication. 
Correct Answer: The correct answers are options a and d. CDS systems can be implemented to monitor patient's health conditions or decrease medicine cost. CDS cannot replace pharmacists to counsel patients or advise health care professionals.

2. Which of the following is not a keyword used to determine included studies of this review?
a. Pharmacist
b. Computerized
c. Clinical
d. Doctor

Correct Answer: The correct answer is option d. This study reviews CDSs used by pharmacists in inpatient/ clinical settings. Doctors are not part of the inclusion criteria.

\section{Protection of Human and Animal Subjects}

There were no human subjects involved in this project.

\section{Conflict of Interest}

None declared.

\section{Acknowledgment}

This study was supported by University of Utah College of Pharmacy. Also, we are very thankful to the editorial board of Applied Clinical Informatics journal for their valuable and constructive comments.

\section{References}

1 Dolovich L, Austin Z, Waite N, et al. Pharmacy in the 21 st century: enhancing the impact of the profession of pharmacy on people's lives in the context of health care trends, evidence and policies. Can Pharm J 2018;152(01):45-53

2 Francis J, Abraham S. Clinical pharmacists: bridging the gap between patients and physicians. Saudi Pharm J 2014;22(06): 600-602

3 Carter BL. Evolution of clinical pharmacy in the USA and future directions for patient care. Drugs Aging 2016;33(03):169-177

4 Saiyed SM, Davis KRKD, Kaelber DC. Differences, opportunities, and strategies in drug alert optimization-experiences of two different integrated health care systems. Appl Clin Inform 2019;10(05):777-782

5 Health IT. Clinical decision support. The Office of the National Coordinator for Health Information Technology. Accessed January 8, 2021 at: https://www.healthit.gov/sites/default/files/page/ 2018-04/Optimizing_Strategies_508.pdf

6 Lainer M, Mann E, Sönnichsen A. Information technology interventions to improve medication safety in primary care: a systematic review. Int J Qual Health Care 2013;25(05):590-598

7 Ibáñez-Garcia S, Rodriguez-Gonzalez C, Escudero-Vilaplana V, et al. Development and evaluation of a clinical decision support system to improve medication safety. Appl Clin Inform 2019;10 (03):513-520

8 Ozkaynak M, Metcalf N, Cohen DM, May LS, Dayan PSMR, Mistry RD. Considerations for designing EHR-embedded clinical decision support systems for antimicrobial stewardship in pediatric emergency departments. Appl Clin Inform 2020;11(04):589-597

9 Wang J, Liang H, Kang H, Gong Y. Understanding health information technology induced medication safety events by two conceptual frameworks. Appl Clin Inform 2019;10(01):158-167
10 Unberath P, Prokosch HU, Gründner J, Erpenbeck M, Maier C, Christoph J. EHR-independent predictive decision support architecture based on OMOP. Appl Clin Inform 2020;11(03):399-404

11 Wright A, Sittig DF, Ash JS, et al. Development and evaluation of a comprehensive clinical decision support taxonomy: comparison of front-end tools in commercial and internally developed electronic health record systems. J Am Med Inform Assoc 2011;18 (03):232-242

12 Lyell D, Magrabi F, Coiera E. Reduced verification of medication alerts increases prescribing errors. Appl Clin Inform 2019;10(01): 66-76

13 Curtain C, Peterson GM. Review of computerized clinical decision support in community pharmacy. J Clin Pharm Ther 2014;39(04): 343-348

14 Zeng X, Zhang Y, Kwong JSW, et al. The methodological quality assessment tools for preclinical and clinical studies, systematic review and meta-analysis, and clinical practice guideline: a systematic review. J Evid Based Med 2015;8(01):2-10

15 Nelson SD, Poikonen J, Reese T, El Halta D, Weir C. The pharmacist and the EHR. J Am Med Inform Assoc 2017;24(01):193-197

16 Saverno KR, Hines LE, Warholak TL, et al. Ability of pharmacy clinical decision-support software to alert users about clinically important drug-drug interactions. J Am Med Inform Assoc 2011; 18(01):32-37

17 Nelson S, Newbold J, Reese T, et al. Computerized clinical decision support systems for clinical pharmacy in the MeaningfulUse Era: a systematic review. National Institute for Health Research. Published 2015. Accessed January 8, 2021 at: https://www.crd. york.ac.uk/PROSPERO/display_record.php?RecordID=16952

18 Chandler J, Higgins JPT, Deeks JJ, Davenport C, Clarke MJ. Chapter 1: Introduction. In: Higgins JPT, Churchill R, Chandler J, Cumpston MS, eds. Cochrane Handbook for Systemic Reviews of Interventions version 5.2.0. 2017:1-11

19 Covidence. Accessed January 8, 2021 at: https://www.covidence. org/

20 Wells G, Shea B, O'Connell D, et al. The Newcastle-Ottawa Scale (NOS) for assessing the quality of nonrandomised studies in metaanalyses. The Ottawa Hospital Research Institute. Published 2019. Accessed January 8, 2021 at: http://www.ohri.ca/programs/ clinical_epidemiology/oxford.asp

21 Aziz MT, Rehman TU, Qureshi S, Andleeb S. Effects of multidisciplinary teams and an integrated follow-up electronic system on clinical pharmacist interventions in a cancer hospital. Int J Clin Pharm 2017;39(06):1175-1184

22 Boussadi A, Caruba T, Karras A, et al. Validity of a clinical decision rule-based alert system for drug dose adjustment in patients with renal failure intended to improve pharmacists' analysis of medication orders in hospitals. Int J Med Inform 2013;82(10):964-972

23 Claus BOM, Colpaert K, Steurbaut K, et al. Role of an electronic antimicrobial alert system in intensive care in dosing errors and pharmacist workload. Int J Clin Pharm 2015;37(02):387-394

24 Cornu P, Steurbaut S, Soštarić S, Mrhar A, Dupont AG. Performance of a clinical decision support system and of clinical pharmacists in preventing drug-drug interactions on a geriatric ward. Int J Clin Pharm 2014;36(03):519-525

25 Díaz A, Saez de la Fuente J, Esteva L, et al. Drug prescribing in patients with renal impairment optimized by a computer-based, semi-automated system. Int J Clin Pharm 2013;35(06): $1170-1177$

26 Eppenga WL, Derijks HJ, Conemans JMH, Hermens WAJJ, Wensing M, De Smet PAGM. Comparison of a basic and an advanced pharmacotherapy-related clinical decision support system in a hospital care setting in the Netherlands. J Am Med Inform Assoc 2012;19(01):66-71

27 Gallagher J, O’Sullivan D, McCarthy S, et al. Structured pharmacist review of medication in older hospitalised patients: a costeffectiveness analysis. Drugs Aging 2016;33(04):285-294 
28 Lightfoot M, Sanders A, Burke C, Patton J. Clinical pharmacist impact on intensive care unit delirium: intervention and monitoring. Hosp Pharm 2019;54(03):180-185

29 Mansour H, Dilkhush D, Lannigan J, Whalen KL. The impact of a computerized potassium alert on adverse drug events and pharmacists' interventions. J Pharm Technol 2010;26(02):55-59

30 McCoy AB, Cox ZL, Neal EB, et al. Real-time pharmacy surveillance and clinical decision support to reduce adverse drug events in acute kidney injury: a randomized, controlled trial. Appl Clin Inform 2012;3(02):221-238

31 Cox Z, Nelsen C, Waitman L, McCoy J, Peterson J. Clinical decision support improves initial dosing and monitoring of tobramycin and amikacin. Am J Health Syst Pharm. 2008;23(01):1-7

32 García-Molina Sáez C, Urbieta Sanz E, Madrigal de Torres M, Vicente Vera T, Pérez Cárceles MD. Computerized pharmaceutical intervention to reduce reconciliation errors at hospital discharge in Spain: an interrupted time-series study. J Clin Pharm Ther 2016;41(02):203-208

33 O'Sullivan D, O'Mahony D, O'Connor MN, et al. Prevention of adverse drug reactions in hospitalised older patients using a software-supported structured pharmacist intervention: a cluster randomised controlled trial. Drugs Aging 2016;33(01):63-73

34 Wilson FP, Shashaty M, Testani J, et al. Automated, electronic alerts for acute kidney injury: a single-blind, parallel-group, randomised controlled trial. Lancet 2015;385(9981):1966-1974

35 Kan T, Kwan D, Chan T, Das P, Raybardhan S. Implementation of a clinical decision support tool to improve antibiotic IV-to-oral conversion rates at a community academic hospital. Can J Hosp Pharm 2019;72(06):455-461

36 Robertson J, Walkom E, Pearson SA, Hains I, Williamsone M, Newby D. The impact of pharmacy computerised clinical decision support on prescribing, clinical and patient outcomes: a systematic review of the literature. Int J Pharm Pract 2010;18 (02):69-87

37 Reese TJ, Kawamoto K, Del Fiol G, et al. When an alert is not an alert: a pilot study to characterize behavior and cognition associated with medication alerts. AMIA Annu Symp Proc 2018: 1488-1497

38 Friedman CPA. A "fundamental theorem" of biomedical informatics. J Am Med Inform Assoc 2009;16(02):169-170 